\title{
THE IMPACT OF LEVEL OF DETAIL IN 3D CITY MODELS FOR CFD-BASED WIND FLOW SIMULATIONS
}

\author{
C.García-Sánchez ${ }^{\mathrm{a}, *}$ S. Vitalis ${ }^{\mathrm{a}}$, I. Pađen ${ }^{\mathrm{a}}$, J. Stoter ${ }^{\mathrm{a}}$ \\ 3D geoinformation group, Urban data science, Faculty of Architecture and Built Environment, Delft University of technology
}

KEY WORDS: Level of Details, Semantic Surfaces, 3D City Models, Computational Fluid Dynamics, Wind Flows, Pedestrian Wind Comfort.

\begin{abstract}
:
Climate change and urbanization rates are transforming urban environments, making the use of 3D city models in computational fluid dynamics (CFD) a fundamental ingredient to evaluate urban layouts before construction. However, current geometries used in CFD simulations tend to be built by CFD experts to test specific cases, most of the times oversimplifying their designs due to lack of information or in order to reduce complexity. In this work we explore what are the effects of oversimplifying geometries by comparing wind simulations of different level of detail geometries. We use semantic 3D city models automatically built and adjust them to their suitable use in CFD. For the first test, we explore wind simulations within a troublesome section of the TUDelft campus, the passage next to the EWI building (the tallest building in our domain), where the use of 3D city model variants show how differences in geometry and surface properties affect local wind conditions. Finally we analyze what these differences in velocity magnitude could mean for practitioners in terms of pedestrian wind comfort.
\end{abstract}

\section{INTRODUCTION}

Climate change and urbanization are pushing the comfort boundaries of our cities. Simulation of such comfort with Computational fluid dynamics (CFD) modelling is therefore an essential part to evaluate urban layouts before construction. Current practices in CFD communities tend to oversimplify the geometries they use, most of the time due to lack of information or in order to reduce complexity. However, the influence of the geometric complexity to the outcome of such simulations have been rarely quantified, so far.

Even if mostly ignored by the CFD community, 3D city models have long before established the concept of levels of detail (LoDs) of the 3D geometric representation of urban areas (Open Geospatial Consortium, 2012) within the geoinformation field. Based on that, building geometry can be modelled with different complexity depending on different application needs. Existing 3D city models can be found to contain buildings in LoD 1 (i.e. prismatic shapes with flat roofs approximating the height of the original building) or LoD 2 (i.e. having simple roof shape). In addition to $\mathrm{LoD}, 3 \mathrm{D}$ city models utilise semantics to denote the different types of objects or surfaces in a urban environment; for instance, buildings, green areas and water bodies are denoted as such in the model. These can be utilised in CFD to approximate their material properties.

In this work we explore the effects of oversimplifying geometries by comparing wind simulations results with different LoD geometries and diverse semantics. To accomplish this goal, we use semantic 3D city models adjusted to their suitable use in CFD, and we predict wind flows in a section of the TU Delft campus using the validated CFD libraries from OpenFOAM. For simplicity, we focus on a unique wind direction for which high wind speeds were experienced within the area in the past. The goal of this work is to evaluate the prediction differences caused by geometries at different LoDs and with diverse se-

\footnotetext{
* Corresponding author
}

mantics, and how these should be taken into account when validating with full scale measurements, which is out of the scope of the present work.

\section{PREPARING THE 3D CITY MODEL FOR CFD SIMULATIONS}

For the research presented in this paper, we concentrate on 4 different cases that are designed around a 3D city model of a part of the TU Delft campus. The geometry used is at two different LoDs: LoD 1.3 block models with multiple extrusion heights in case of a significant height jump (like a church with a tower); and LoD 2.2 containing roof structures (Figure 1), see the improved LoD specifications for 3D building models (Biljecki et al., 2016).

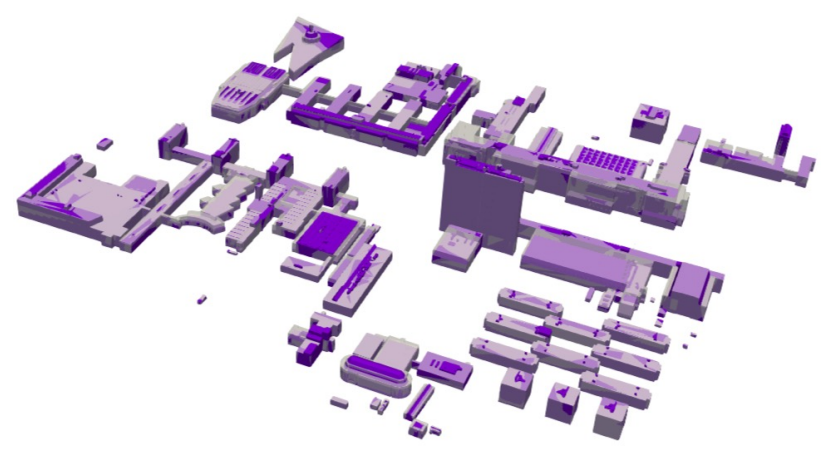

Figure 1. LoD1.3 (grey with transparency) and LoD2.2 (purple) geometry comparison.

The 3D city model geometries were downloaded directly in tiles from the 3D BAG database (Dukai et al., 2021). This database contains 3D models at LoD 1.2, 1.3 and 2.2 for all 10 million buildings in The Netherlands. Then, we used the open-source software cjio (Ledoux et al., 2019) to extract the buildings of our test area and reduce the extension of our domain (since the full tiles were to large for the current CFD study). 
In addition to the use of the geometries in Computational Fluid Dynamics, we also explore advanced capabilities of 3D city models, such as the use of semantics (see Figure 2 with semantics in LoD 2.2). Specifically, we focus on analyzing the impact of water and green surfaces in our simulations. In order to assess the impact of water and vegetation we introduce them as surfaces based on the semantic classification deriving from the Key register Large-scale Topography (in Dutch: Basisregistratie Grootschalige Topografie, BGT).

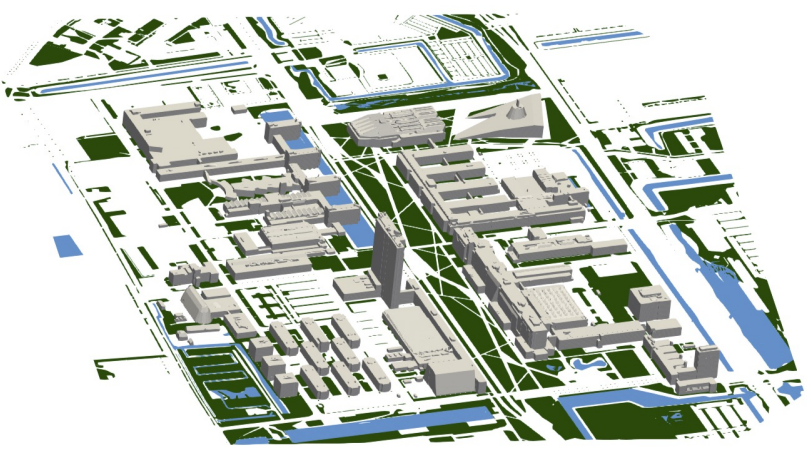

Figure 2. LoD2.2 with semantic surfaces for water (blue) and vegetation (green)

For this, we downloaded an extract of the BGT for a greater area of Delft from www.PDOK.nl, the Dutch national geoportal. Using QGIS, we clipped the data based on a polygon that defines the area of interest; the latter derived by computing the oriented bounding box that contains the aforementioned buildings and enlarging it (using a buffer zone) by $2 \mathrm{~km}$. Finally, we used BlenderGIS to convert the data to a format appropriate for further analysis (such as .stl or .obj formats).

Table 1 summarizes the different test cases ran to investigate the impact of two different geometrical LoDs (i.e. LoD1.3 and LoD2.2) as well as the use of water and vegetation in the CFD wind-simulation:

\begin{tabular}{cccc}
\hline Case & LoD & \multicolumn{2}{c}{ Semantic surfaces } \\
\hline & & water & vegetation \\
1 & 1.3 & no & no \\
2 & 2.2 & no & no \\
3 & 2.2 & yes & no \\
4 & 2.2 & yes & yes \\
\hline
\end{tabular}

Table 1. Different wind simulations performed within the study.

\section{WIND SIMULATIONS SET-UP}

To perform the CFD simulations we use the open-source libraries OpenFOAM, version 7 (The OpenFOAM Foundation, 2021).

\subsection{Governing equations}

To complete the simulations we use the Reynolds-averaged Navier-Stokes (RANS) approach for steady incompressible flows. The equations that govern the flow are the following:

$$
\frac{\partial \overline{u_{j}}}{\partial x_{j}}=0
$$

$$
\overline{u_{j}} \frac{\partial \overline{u_{i}}}{\partial x_{j}}=-\frac{1}{\rho} \frac{\partial \bar{p}}{\partial x_{i}}+\nu \frac{\partial^{2} \overline{u_{j}}}{\partial x_{j} \partial x_{j}}-\frac{\partial \overline{u_{i}^{\prime} u_{j}^{\prime}}}{\partial x_{j}}
$$

where $\overline{u_{i}}$ are time-averaged velocity components, $\rho$ is the density, $\bar{p}$ the pressure and $\nu$ is the kinematic viscosity. The term $\overline{u_{i}^{\prime} u_{j}^{\prime}}$ represents the Reynolds stress tensor, which is unknown and needs to be closed with a turbulence model. For our case we used the two equations $k-\varepsilon$ turbulence model (Wilcox, 1993) since it is a widely used model in outdoor wind simulations and it is a rather simple model. In this turbulence model, the Reynolds stress tensor is computed using a linear eddy viscosity approach where the turbulent viscosity is computed following equation 3:

$$
\nu_{t}=0.09 \frac{k^{2}}{\varepsilon}
$$

Lastly, the equations for the two turbulence variables, $k$ and $\varepsilon$ are as follows:

$$
u_{j} \frac{\partial k}{\partial x_{j}}=\frac{\partial}{\partial x_{j}}\left[\left(\nu+\frac{\nu_{t}}{\sigma_{k}}\right) \frac{\partial k}{\partial x_{j}}\right]+P_{k}-\varepsilon
$$

$$
u_{j} \frac{\partial \varepsilon}{\partial x_{j}}=\frac{\partial}{\partial x_{j}}\left[\left(\nu+\frac{\nu_{t}}{\sigma_{\varepsilon}}\right) \frac{\partial \varepsilon}{\partial x_{j}}\right]+C_{\varepsilon 1} \frac{\varepsilon}{k} P_{k}-C_{\varepsilon 2} \frac{\varepsilon^{2}}{k}
$$

where $P_{k}$ is the turbulent production term and $\sigma_{k}, \sigma_{\varepsilon}, C_{\varepsilon 1}$ and $C_{\varepsilon 2}$ are model constants with the corresponding values: 1.0 , $1.3,1.44$ and 1.92 .

\subsection{Computational domain and mesh}

The computational domain is the extension of air around the buildings modelled within the CFD simulation. This extension is based on best practice guidelines, ensuring that domain boundaries pose a negligible numerical effect in the solution within the area of interest (Franke et al., 2007). Thus, the domain extends for approximately $2 \times 2.7 \mathrm{~km}^{2}$ in the horizontal direction, and approximately $600 \mathrm{~m}$ in the vertical direction. These dimensions correspond to multiples of the highest building in the test area $(H)$, which is approximately $98 \mathrm{~m}$ tall (here on referenced as EWI building, the Faculty of Electrical Engineering, Mathematics and Computer Science). The semantic surfaces used for the different cases are selected applying a similar approach, by clipping the surfaces $100 \mathrm{~m}(\sim 1 H)$ away from the buildings outline.

Before running the wind simulations, we need to create the mesh which discretizes the space around the buildings where the airflow is modelled. To complete this task we used the automatic parallel mesh generator snappyHexMesh.

The resulting meshes contain mostly hexahedras and some tetrahedras, with approximately 16 million cells for all cases. The cell density increases closer to building and semantic surfaces as seen in Figure 3.

\subsection{Boundary conditions}

For the current analysis we focus on one main wind direction, since it is known for causing high, hindering wind speeds in 


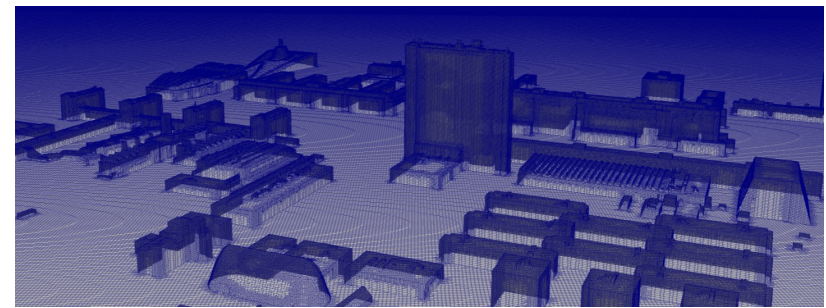

Figure 3. LoD2.2 mesh snapshot.

the vicinity of a tall building within the area (EWI building). This wind direction corresponds to an angle of $45^{\circ}$ with north, with wind blowing from south-west direction. Other authors already studied this wind direction and magnitude by numerically investigating the effect of vegetation within the area to alleviate the strongest wind accelerations (Kenjereš and ter Kuile, 2013). To define the velocity magnitude we used the available meteorological wind measurement data for the period JanuaryOctober of 2019, at the Rotterdam Station (Koninklijk Nederlands Meteorologisch Instituut (KNMI), 2019), a city close to Delft. The measurements were performed at a height of $10 \mathrm{~m}$, and the data, which is averaged along the period of time considered to the amount of $4.9 \mathrm{~m} / \mathrm{s}$, is introduced in our model at the same height.

We assumed the atmospheric boundary layer stratification is neutral, thus neglecting any temperature forcing. Consequently, the simulations used the characteristic neutral logarithmic profile at the inlet, where the velocity is computed as:

$$
U=\frac{u_{*}}{\kappa} \ln \left(\frac{z+z_{0}}{z_{0}}\right),
$$

Since we model turbulence with the standard $k$ - $\varepsilon$ turbulence model, we set the inflow turbulent variables, $k$ and $\varepsilon$, following their consistent formulation through equations 7 and 8 :

$$
\begin{gathered}
k=\frac{u_{*}^{2}}{\sqrt{C_{\mu}}}, \\
\varepsilon=\frac{u_{*}^{3}}{\kappa\left(z+z_{0}\right)},
\end{gathered}
$$

where, $\kappa$ is the von Karman constant set to 0.41 , and $u_{*}$ is the friction velocity computed by the software. We set the roughness length for the terrain, $z_{0}$, to $0.5 \mathrm{~m}$ corresponding to a 'very rough' area with scattered buildings (Wieringa, 1992). In addition, the roughness length for water and vegetation correspond to the values of $0.0002 \mathrm{~m}$ and $0.03 \mathrm{~m}$, respectively. It is important to mention that trees were not considered in this study, and the same vegetation type was assumed in all the area. These roughness length values were used within the rough wall function based on $z_{0}$ used at the ground patches (Parente et al., 2011). For the buildings walls we applied a smooth wall function.

To run the simulation we used the simpleFoam solver with blended second order schemes. The simulations were run for 3000 iterations reaching residuals in the order of $10^{-5}-10^{-6}$.

\section{RESULTS}

The results are presented through contour plots for wind speed and turbulent kinetic energy at pedestrian height $(1.75 \mathrm{~m})$.

\subsection{Velocity magnitude}

Figure 4 introduces the velocity magnitude for the four cases we are considering. The color map is normalized to the inflow velocity to improve visualization. A priori we observe the largest differences for cases 1 (LoD 1.3) and 4 (LoD 2.2 with water and vegetation), which is expected, since they are geometrically most different.
LoD1.3

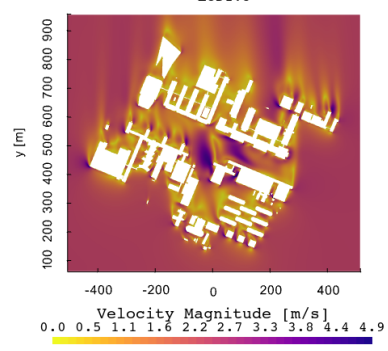

LoD2.2 with water

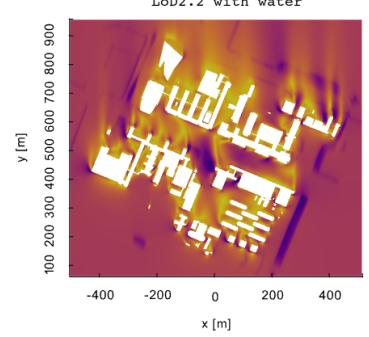

LoD2.2
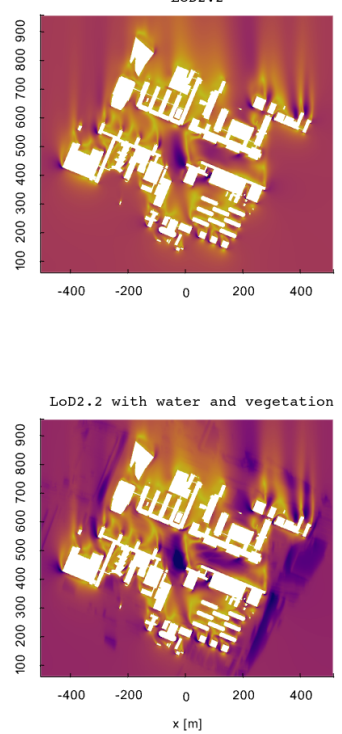

Figure 4. Velocity magnitude for the four cases at pedestrian height $(1.75 \mathrm{~m})$.

However, to better understand the deviations from the diverse geometries we include Figure 5, where we plot the relative differences between the LoD 2.2 case (case 2) and the other three cases at pedestrian height. To normalize to a relative value we used the inflow velocity at the same height which was approximately $2.54 \mathrm{~m} / \mathrm{s}$.

There are a few observations that we can enumerate for each case:

- Differences between LoD 2.2 and LoD 1.3: we can see localized differences in the velocity. The maximum difference in wind speed is typically found closest to tall buildings, but we also see noticeable deviations caused by low buildings differences in bottom left locations. Overall most times there is an increase in velocity related to the usage of the higher detail of the LoD2.2 model. This could be related to the better surface representation which deviates from a rough sharp square edge in comparison the lower LoD1.3 description.

- Differences between LoD 2.2 and LoD 2.2 with water: contrary to the previous case, here we observe a consistent lower velocity when the water is not included within the geometry. This aligns with the surface roughness implemented, which is lower for the water, thus facilitating the acceleration of wind close to the surface. 

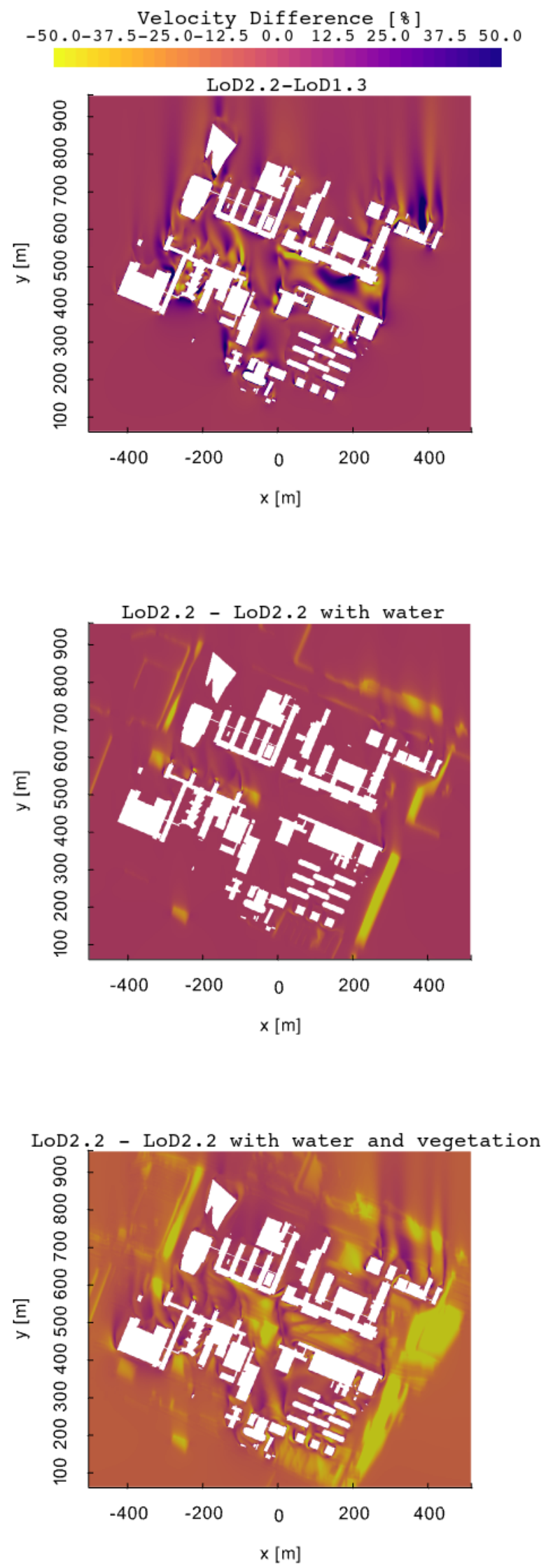

Figure 5. Velocity magnitude differences at $1.75 \mathrm{~m}$ height.
- Differences between LoD 2.2 and LoD 2.2 with water and vegetation: similarly to the previous case, here we see a consistent lower velocity at different scales than when the water and vegetation are not included within the geometry. Which again aligns with using different surface roughness values for the water and vegetation patches.

Overall, looking at the previous two figures we see that including the current water and vegetation patches increases the local wind speed at pedestrian height. This is especially relevant in the passages within buildings in the area (specially next to the EWI building), where we can observe the highest wind speed with LoD 2.2 with water and vegetation. This shows that using LoD 2.2 instead of LoD 1.3 and including water and vegetation does make a difference and results in a more realistic case, since accelerations in that passage has been observed in the past.

\subsection{Turbulent kinetic energy}

To further support previous velocity results we also included the results obtained for turbulence. In Figure 6 we introduce the turbulent kinetic energy at $1.75 \mathrm{~m}$ height for the four cases studied. It can be seen how the largest differences still hold between case 1 and case 4 , however the differences are somewhat less noticeable for the turbulent kinetic energy than for the velocity magnitude.
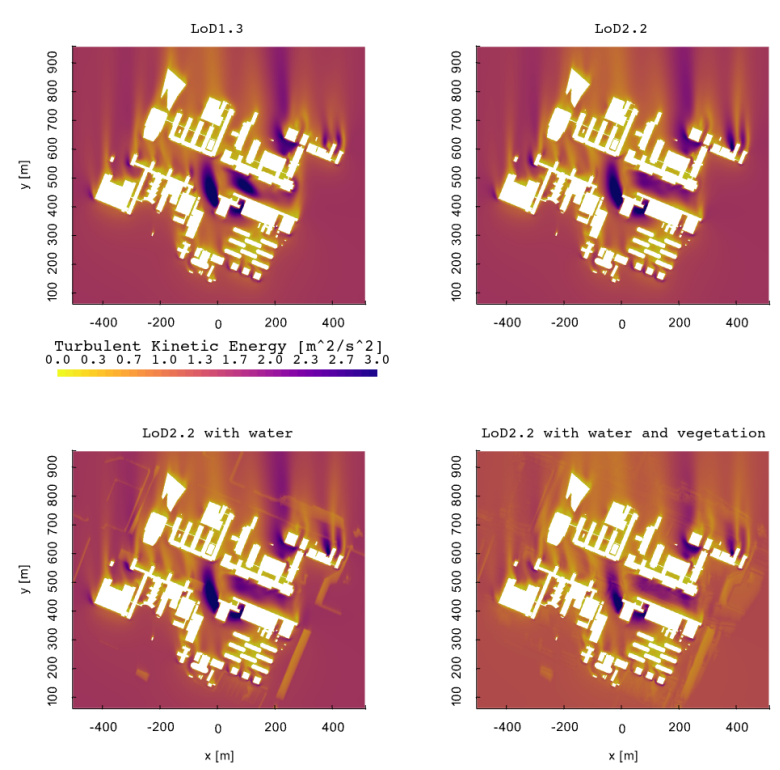

Figure 6. Turbulent kinetic energy for the four cases at pedestrian height $(1.75 \mathrm{~m})$.

In all contours, turbulent kinetic energy values rise in locations where large flow separations take place, while they decrease in free building locations where path roughness diminishes. Consequently, we can highlight the fact that the turbulent kinetic energy for case 4 is overall lower than for the other three cases. In addition, the area with higher turbulence next to the tallest building within the domain is smaller for the fourth case as well.

\subsection{Pedestrian wind comfort}

The reason why wind speed differences may matter from a city design perspective resides in thresholds created to build urban 
areas that accommodate the people living within. Pedestrian wind comfort guidelines were created for this purpose, and although several references have been published in the past, here we analyze what our results mean within the comfort criteria for the City of London (of London Corporation, 2019). The following Table 2 summarizes the different categories for this specific wind comfort criteria:

\begin{tabular}{lcc}
\hline Category & $\begin{array}{c}\text { Mean \& GEM } \\
\text { wind speed }\end{array}$ & $\begin{array}{c}\text { Max. exceedance } \\
\text { probability }\end{array}$ \\
\hline frequent siting & $2.5 \mathrm{~m} / \mathrm{s}$ & $5 \%$ \\
occasional siting & $4 \mathrm{~m} / \mathrm{s}$ & $5 \%$ \\
standing & $6 \mathrm{~m} / \mathrm{s}$ & $5 \%$ \\
walking & $8 \mathrm{~m} / \mathrm{s}$ & $5 \%$ \\
uncomfortable & $>8 \mathrm{~m} / \mathrm{s}$ & $5 \%$ \\
unsafe & $15 \mathrm{~m} / \mathrm{s}$ & $0.022 \%$ \\
\hline
\end{tabular}

Table 2. Wind comfort categories, simplified from (of London Corporation, 2019). GEM: Gust equivalent mean

Using this wind comfort we created Figure 7, where the results at $1.75 \mathrm{~m}$ height are plotted in function of the pedestrian comfort classification. Considering that the inflow velocity magnitude was set to $4.9 \mathrm{~m} / \mathrm{s}$ it is expected not to retrieve sections of the urban area within the highest wind speed classifications.
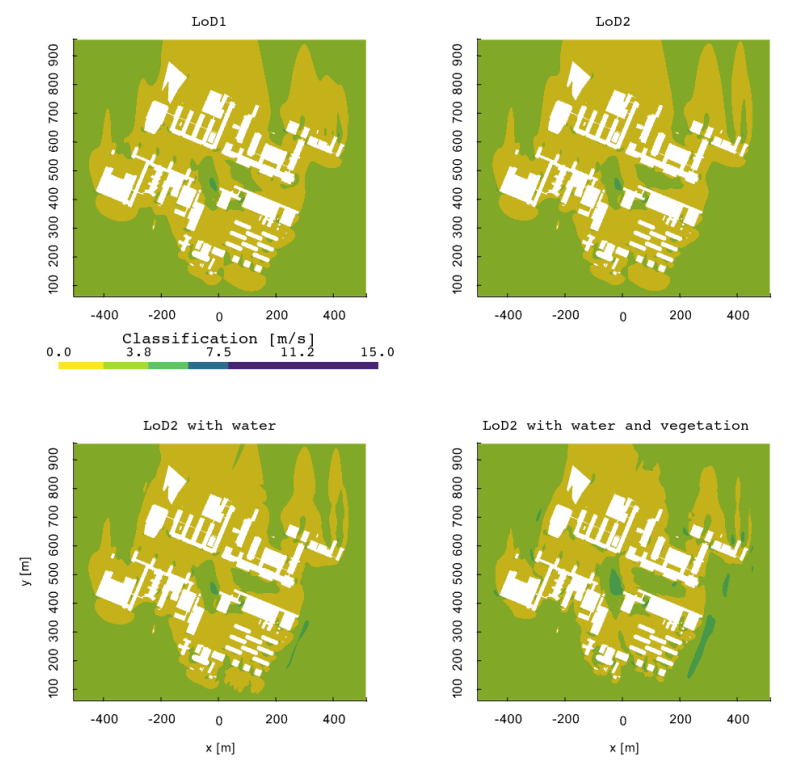

Figure 7. Pedestrian wind comfort classification at $1.75 \mathrm{~m}$ height.

However, we can observe how the contour plots differ between the four cases, specially between case 1 and case 4 . Some of the consequences that these differences would imply in practice could affect decisions such as the placement of restaurant terraces, outdoor sitting and balconies, since in those locations sitting ranges from frequent to occasional. In addition, specifically for case 4 we can observe two larger areas (which are close to tall buildings) for which velocities within 4 and $6 \mathrm{~m} / \mathrm{s}$ were retrieved. This means that not even occasional sitting would be recommended here, and only standing activities could be allowed, for example.

\section{CONCLUSIONS}

Within this short article we presented a numerical analysis over four cases with diverse geometrical and semantic configurations. We compare the results in terms of velocity magnitude and turbulent kinetic energy, further deriving their classification within pedestrian wind comfort criteria.

We can conclude that different levels of detail lead to diverse wind patterns in built environments. Evidence for this was already presented by other authors through specific cases (Ricci et al., 2017). Here we generalize by using the LoD definition for 3D city models instead, analyzing the geometry impacts through a structured analysis. The results are relevant for wind flows, but they might be relevant for pollution or pathogens transport as well. Further simulations including pollutant transport are the next scope of the current research.

\section{ACKNOWLEDGEMENTS}

We would like to acknowledge Ravi Y. Peters for their help producing the terrain patches for this extended abstract. We are also grateful to the computational resources provided by the INSY cluster (TUDelft) and the support by the Delft Technology fellowship (TUDelft) that made this research possible. The work presented in this paper has received funding from the European Research Council (ERC) under the European Union's Horizon 2020 Research \& Innovation Programme (grant agreement no. 677312 UMnD: Urban modelling in higher dimensions).

\section{REFERENCES}

Biljecki, F., Ledoux, H., Stoter, J., 2016. An improved LOD specification for 3D building models. Computers, Environment and Urban Systems, 59, 25-37.

Dukai, B., van Liempt, J., Peters, R., Stoter, J., Vitalis, S., Wu, T., 2021. 3dbag. https://3dbag.nl/en/viewer.

Franke, J., Hellsten, A., Schlünzen, H., Carissimo, B., 2007. Best practice guidelines for the cfd simulations of flows in the urban environment. Technical report, COST Action 732.

Kenjereš, S., ter Kuile, B., 2013. Modelling and simulations of turbulent flows in urban areas with vegetation. Journal of Wind Engineering and Industrial Aerodynamics, 123(3-55).

Koninklijk Nederlands Meteorologisch Instituut (KNMI), 2019. Climatological data from KNMI. https://www.knmi.nl/nederland-nu/klimatologie/uurgegevens.

Ledoux, H., Arroyo Ohori, K., Kumar, K., Dukai, B., Labetski, A., Vitalis, S., 2019. CityJSON: a compact and easy-to-use encoding of the CityGML data model. Open Geospatial Data, Software and Standards, 4(1).

of London Corporation, C., 2019. Wind microclimate guidelines for developments in the city of london. https://www.cityoflondon.gov.uk/services/environment-andplanning/planning/design/Documents/city-of-london-windmicroclimate-guidelines.pdf.

Open Geospatial Consortium, 2012. City Geography Markup Language (CityGML) Encoding Standard, version: 2.0.0. 
Parente, A., Gorlé, C., van Beeck, J., Benocci, C., 2011. Boundary Layer Meteorology. A comprehensive modelling approach for the neutral atmospheric boundary layer: consistent inflow conditions, wall function and turbulence model, 140(28).

Ricci, A., Kalkman, I., Blocken, B., Burlando, M., Freda, A., Repetto, M., 2017. Local-scale forcing effects on wind flows in an urban environment: Impact of geometrical simplifications. Journal of Wind Engineering and Industrial Aerodynamics, 170, 238-255.

The OpenFOAM Foundation, 2021. Openfoam v7 user guide. Technical report, OpenFOAM.org.

Wieringa, J., 1992. Updating the Davenport roughness classification. Journal of Wind Engineering and Industrial Aerodynamics, 41(44).

Wilcox, D., 1993. Turbulence modeling for CFD. DCW Industries Inc., La Cañada. 